\title{
SEED POINT SELECTION OF SEGMENTATION OF LUNG IN HRCT IMAGES
}

\author{
V.Arun, D.Laxma Reddy \\ Dept. of Electronics and Communication Engineering, \\ MLR Institute of Technology, \\ Hyderabad, Telangana, India
}

\begin{abstract}
This article proposes a completely mechanized calculation for the exact division of high-resolution computerized tomography regions (HRCTs). A portrayal in light of the power of the locale permits considering the picture for the most part comprising of three districts: the base of the $\mathrm{CT}$, the lungs and the chest area encompassing the lungs. Another technique in view of the surge fill calculation is utilized to viably recognize the encompassing district. This progression encourages the utilization of another quick strategy to evacuate the CT foundation utilizing direct outputs beginning from installed pixels. The related segments speaking to the tracheal parts are expelled by watching the partition of the mean and standard deviation of the power esteems between the trachea and the lungs. Divided aspiratory pictures are additionally improved to reestablish pixel force esteems to bronchi and lung restrict. The proposed system isn't just computational easily, yet in addition powerful and precise in distinguishing lung limits. This article introduces a total picture, including illustrations and test comes about.
\end{abstract}

Keywords: Computer-Aided Diagnosis; HRCT lung images; Image analysis; Segmentation; flood-fill method; Image dilation;

\section{INTRODUCTION:}

High-resolution computerized tomography (HRCT) is utilized as a non-intrusive apparatus to analyze and assess lung infection. HRCT lung pictures demonstrate significantly more data than straightforward X-beams, however, require extra aptitudes for translating and removing obsessive data. With a specific end goal to enhance the effectiveness and exactness of lung ailment location, PC helped to find (computer-aided design) frameworks help radiologists to describe the appropriation of malady designs found in high-determination TC pictures. The aspiratory division is the principal part of these computer-aided design frameworks. Lung division is likewise required in the pre-handling of examination and perception devices [1-4].

This article proposes another technique for a quick ID of thoracic areas encompassing the lungs and the CT foundation strategy by filling where the seed spot is consequently decided. Picture zones that contain lungs and bronchi are additionally treated to

evacuate the principle bronchus and upgrade the framework of the lung. The record is sorted out as takes after. Segment II portrays foundation examinations identified with the zone of a pneumonic division. Area III depicts the proposed strategy for the aspiratory division. Segment IV introduces the outcomes and represents the focal points and restrictions of the technique. Area $\mathrm{V}$ gives a synopsis of the work and conceivable future introductions of the exploration.

\section{BACKGROUND}

There are a few PC helped strategies that are proposed and created to portion the lung areas on the chest CT pictures. The 3-
D district development strategy is utilized to locate the left lung and right edge, with the physically appointed seed point. The 2D board following strategy was utilized to discover lung limits [5]. The cut-off strategy has been accomplished for the ideal division all around in measurement $\mathrm{N}$. The strategies displayed in all

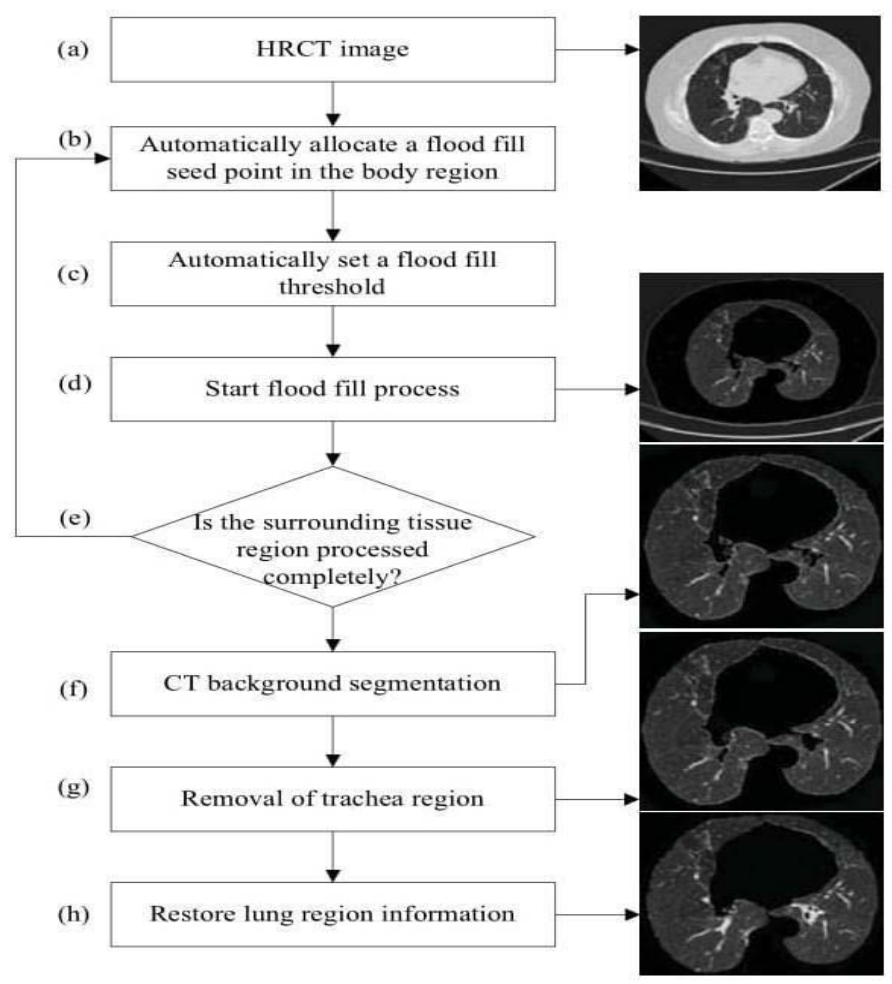

Figure 1. The flow chart of the proposed method. 
require seed filet start manuals. In, the front and back intersection lines are physically created to isolate the left and right lungs [6].

In this paper, we base our technique for the programmed division of all around characterized qualities of a few locales of the aspiratory HRTC force pictures, and utilize the Stream Line Checking Fill Strategy and distinguish the correct operations morphological district. The technique additionally guarantees that each interior pixel in the treated and removed lung area has a similar power an incentive as the first picture.

Aspiratory imaging is a key advance for most pneumonic imaging applications. HRCT pictures have been utilized for applications, for example, pneumonic parenchyma thickness examination for aviation route investigation and illness location. In this exploration HRCT, aspiratory pictures are utilized to identify and break down modules. Most computer-aided design frameworks have two stages, the first is a division of the lung, and the second is to portray the infection design. This lung picture was performed by the manual division of the lung [7-8].

\section{METHODOLOGY}

This area depicts the proposed technique for fragmenting lung areas in HRCT pictures. Figure 1 demonstrates a stream diagram comprising of seven primary advances utilized as a part of the technique. The accompanying is an outline of these means.

Utilizing an HRCT lung picture parcel in $16 \times 16$ boxes, a spot semen position for the full fill calculation is naturally situated in the body locale.

The surge filling process begins with preset limit esteem.

Surge fill technique visits all pixels in an associated body part.

$\square \quad$ A test is performed to check whether the fill has totally gone to the encompassing tissue district. On the off chance that the encompassing tissue isn't totally handled, another surge fill point is naturally appointed to the untreated encompassing

tissue locale and the surge filling procedure will continue.

$\square \quad$ The area relating to the CT foundation is presently erased utilizing an iterative calculation.

Non-lymphatic parts are separated from the base of the moderate aspiratory locale extricated in its mean and deviation from the pixel force.

$\square \quad$ The lung locale and the aviation route district can be recognized by their distinction in pixel force. A $3 \times 3$ even and vertical dilatation veil is connected to recoup missed lung data amid the surge filling process.

\section{A. HRCT image}

High-determination registered tomography is

a kind of processed tomography (CT) with particular methods to upgrade picture determination. It is utilized as a part of the analysis of different medical issues, however most generally for lung sickness, by evaluating the lung parenchyma.
Mechanized tomography (CT) is as of now the best imaging technique for diagnosing lung

illness. High-determination CT scanners produce a threedimensional perspective of organs with symmetric determination pictures in pivotal segments. It gives a point by point data on lung parenchyma and can layout structures up to the optional lumbar projection level, the littlest lung structure. It is especially helpful for the conclusion of pictures, since the changed life structures of the lungs, caused by an infection, can be unmistakably found in a thin CT picture.

\section{Method:}

HRCT is performed utilizing a customary CT scanner. Be that as it may, picture parameters are boosted spatial determination.

Slight cut width (normally 1-2 mm) is utilized.

A high-determination spatial determination picture calculation.

The visual field is limited to limit the span of every pixel. Other output factors (eg Point of convergence) can be upgraded for determination to the detriment of filtering speed.

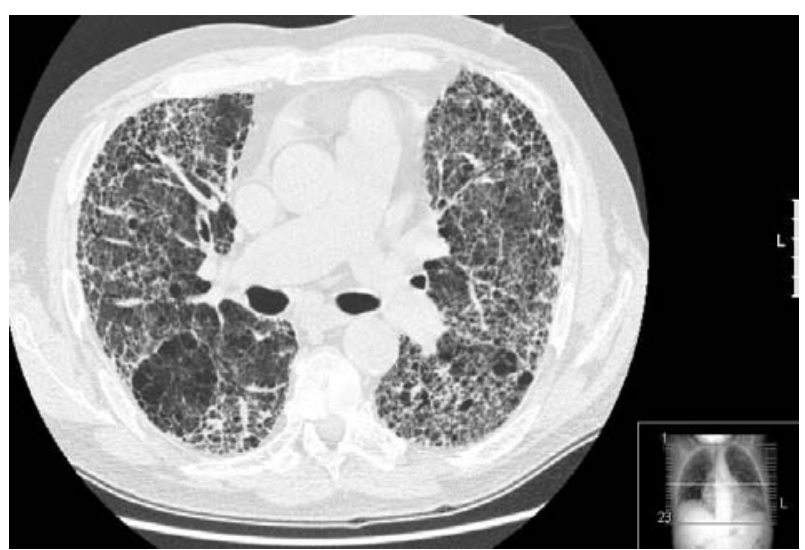

Fig 2: High-resolution computed tomography (HRCT)

\section{B. Automatic Seed Point Selection}

As found in the past segment, the thoracic area of the chest is situated between the CT's fundus and the lung district in HRCT pneumonic pictures. The usage of the surge fill strategy in the encompassing tissue district would then be able to isolate the CT foundation and the aspiratory area. The siting point is characterized as the point with

the most extreme power in a $16 x 16$ pixel neighborhood with force esteems in the upper area of Figure 2. A 16x16 window was found to satisfactorily portray the practically uniform dissemination of force in an area and furthermore, gives the normal power inside the window. 


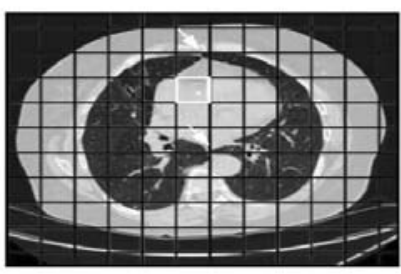

(a)

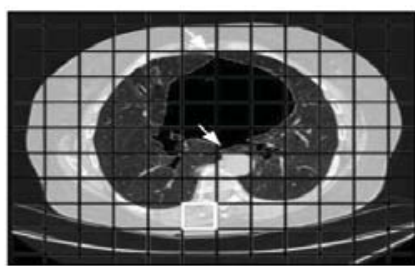

(b)

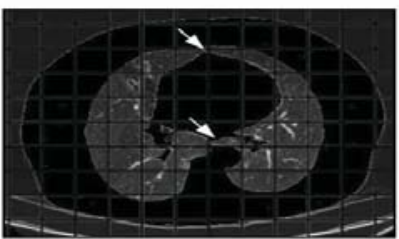

(c)

Figure 3. (a) First seed point allocation. (b) Second seed point allocation. (c) Identification of the complete surrounding tissue region using flood fill.

\section{CT background segmentation}

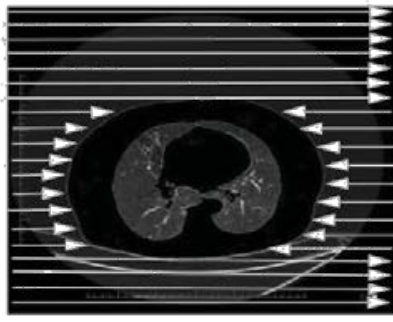

(a)

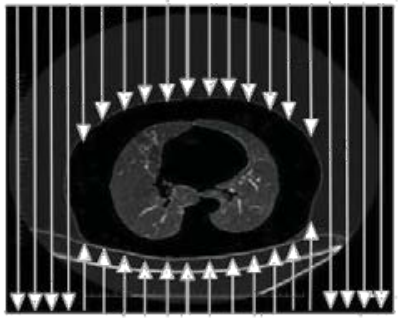

(b)
Figure 4. (a) Horizontal scan. (b) Vertical scan.

In the wake of denoting the whole body district encompassing the lungs, the following assignment is to recognize the outside of this area, which is the CT foundation. This is finished by beginning a straight output from each edge pixel to its association to the inverse edge, denoting every pixel, and halting examining while experiencing a pixel having a place with the encompassing area. Since in the broadest case the encompassing locale has a non-curved shape, it is important to perform flat examining (left and right pixels) and vertical filtering (from the best and base pixels) to totally expel every outer pixel having a place with the base CT. The procedure is outlined in Fig. 4.

Presently we simply need to concentrate on the area of the thick chest situated inside the checked locale of the body. All other data will be erased. Figure 5 demonstrates the thick chest district, which contains respiratory tract (trachea and bronchi) and lungs.

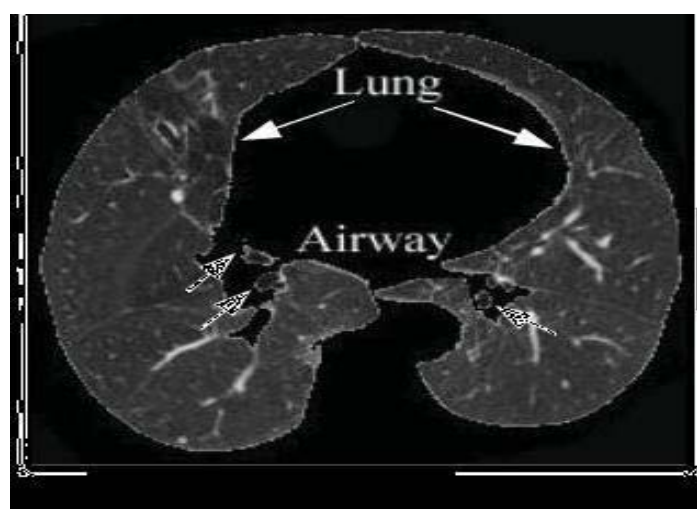

Figure 5: Coarse lung region $\mathbf{D}$.

\section{Removal of trachea region}

Various creators have created procedures for distinguishing lung aviation routes from CT lung pictures. Hu et al. utilized cut bycut area developing technique to expel aviation routes and utilized the aviation routes area on current CT cut to appraise the aviation route's area on the following cut. Nonetheless, the cut by-cut district developing strategy isn't equipped for fragmenting the aviation routes on some transverse cuts.
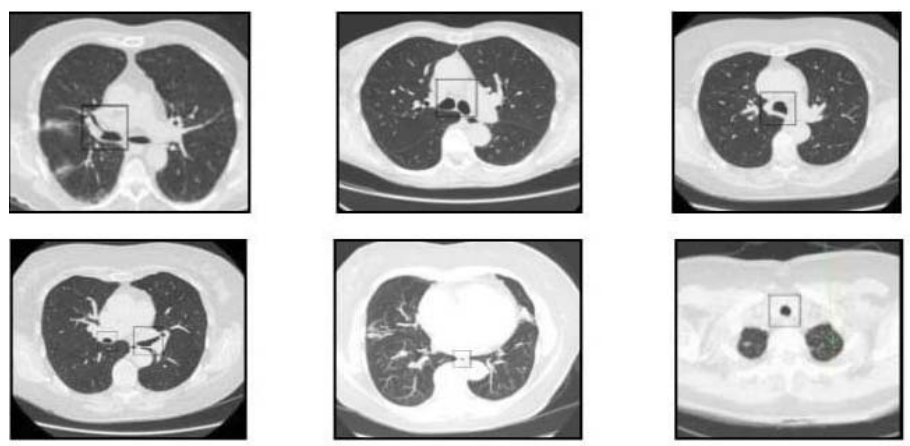

Fig 6:Air filled regions vary in size

\section{E. Restoring lung region information}

Bronchial dividers, veins, and knobs have by and large higher power contrasted with the normal lung area, and in the event that they are close to the limit of the lung locales, they will doubtlessly get evacuated by the surge fill process. In Fig. 8 (b), the lung knob close to the limit is lost in the wake of extricating the coarse lung locale. Reestablishing the lost pixel data inside the lung limit is vital for the legitimate division. We propose a strategy underneath for precise recreation of the lung picture area. 


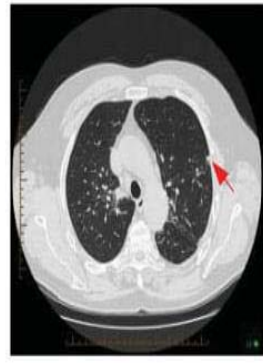

(a)

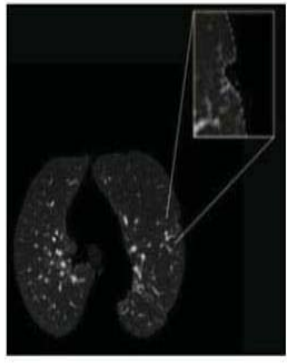

(b)
Figure 8. (a) Lung nodule on the side of the lung boundary. (b) Pixels on the lung nodule affected by the flood fill process.

\section{1) Morphological dilation:}

In grayscale morphology, the operation of enlargement is utilized for locale developing. This operation additionally closes gaps and holes inside the area. Expansion makes a question bigger by including pixels around its edges. Fig. 9

demonstrates a $3 \times 3$ level and vertical expansion cover.

\section{1}

\section{1}

\section{1}

Figure 9: Horizontal and vertical mask

\section{2) Lung boundary enhancement}

In Fig. 12, the dim cells amidst the extended window speak to the pixels from the encompassing tissue area that was duplicated from the first picture to the lung limit. The cells set apart with numbers are pixels in the lung locale, and the dark cells relate to the foundation pixels. A sweep line calculation is utilized to discover the edge pixels which are adjoining the dark foundation pixels. Each edge pixel has a foundation pixel incorporated into its 8 neighboring pixels and is likewise not some portion of the lung district. Each output line peels off edge pixels found on it by setting their pixel power to zero. Rehashing the procedure four times evacuates the 4-pixel wide fringe presented by the enlargement operation.

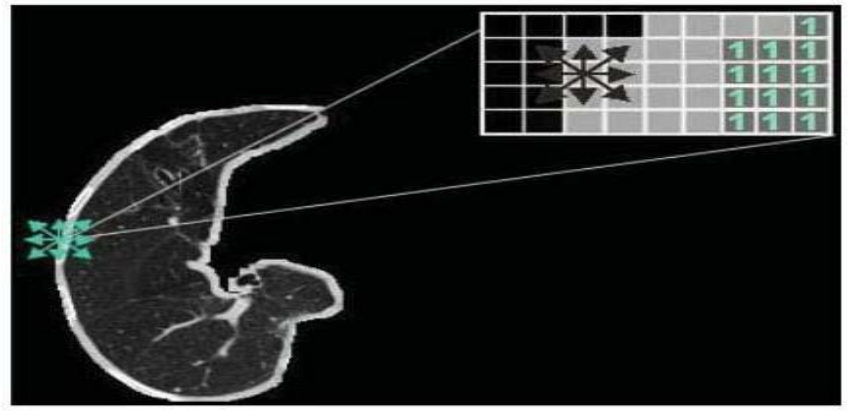

Figure 12. Optimize lung boundary.

\section{EXPERIMENTAL RESULTS AND ANALYSIS}

A figure demonstrates the outcomes after lung division for a set containing unmistakably extraordinary pictures and highlights. In every one of the cases, the lung district has been effectively sectioned. Keeping in mind the end goal to demonstrate the power and exactness of the proposed strategy, the lung districts in the test pictures have fluctuating shape, size, and area. As observed from the outcomes, aviation routes areas of various sizes and shapes have additionally been identified and evaluated by the proposed strategy.

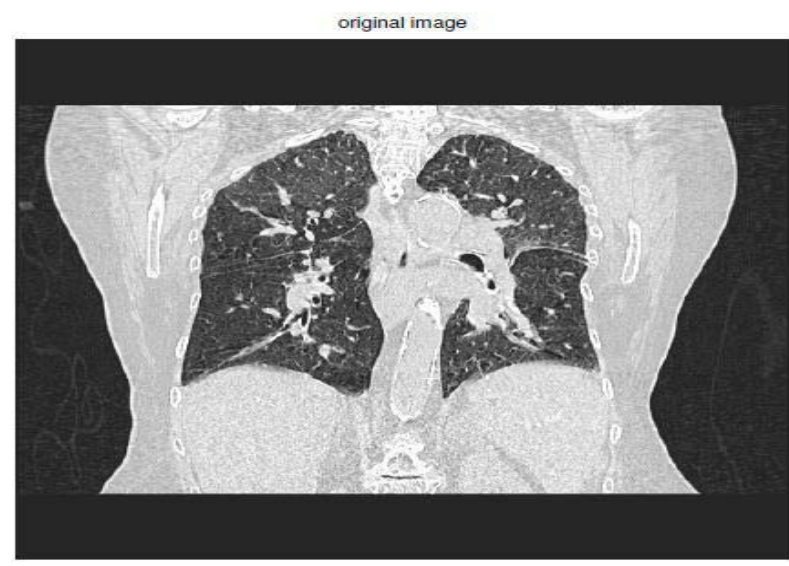

Fig 10. Original image

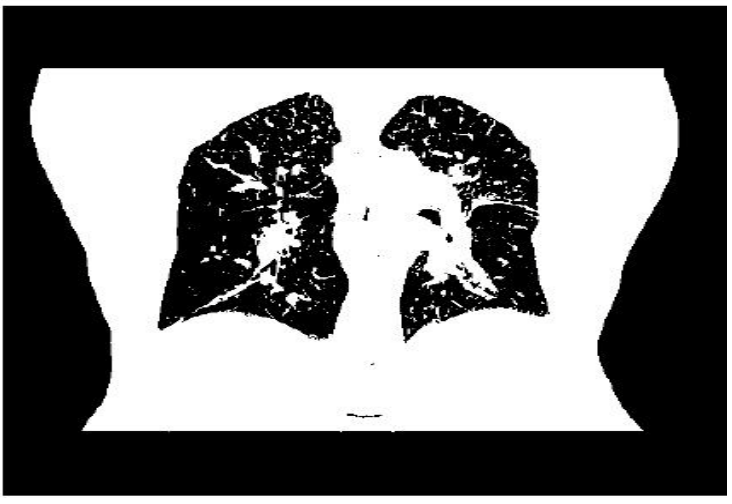

Fig 11. Flood fill threshold

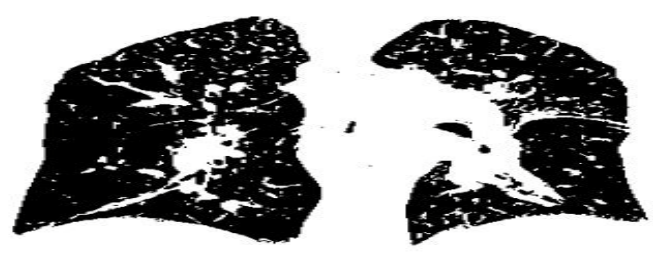

Fig 12. Flood fill process 


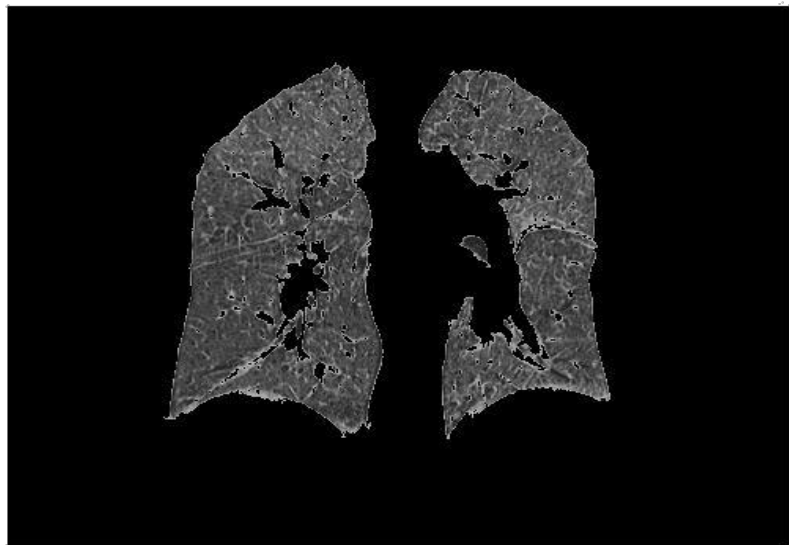

Fig 13. Trachea region \& filling holes

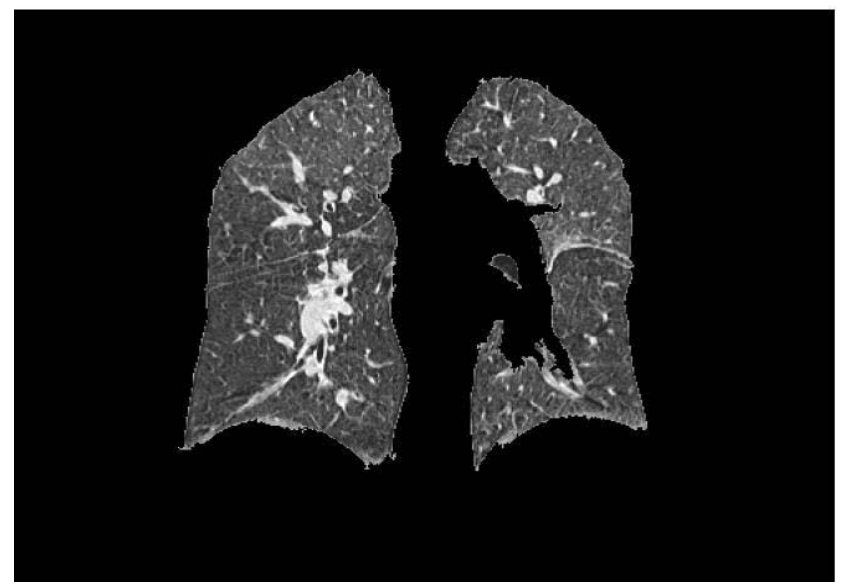

Fig 14. Lung region information

\section{A) Advantages of the proposed method}

The strategy is completely programmed; it doesn't require manual contribution of seed focuses or intelligent choice of districts

$\square \quad$ The strategy gives the exact division of the lung area and adequately evacuates the trachea

$\square \quad$ The pixel power esteems inside the inside of the lung locale after division is the same as the first picture.

\section{B)Limitations of the proposed method}

$\square$ The strategy includes a few phases of handling.

\section{CONCLUSION AND FUTURE WORK}

This paper displayed a computerized aspiratory division technique in view of flooding and morphological operations to adequately section the variable dimensional lung locales, shape, and position of HRCT pictures. Utilizing the factual attributes of the force circulation, you can without much of a stretch recognize the lung and aviation route. The exploratory outcomes acquired utilizing a vast accumulation of HRCT pictures demonstrate that the proposed technique is exceedingly viable. For the future change, we plan to enhance the framework by enhancing the filled filling calculation to recognize the chest district, limiting the quantity of examinations utilized as a part of sectioning CT history and taking out rehashed morphological operations. The proposed technique could be enhanced amid fasting by making a pile of HRCT pictures with 3D morphologic administrators.

\section{REFERENCES}

[1] N. A. Memon, A. M. Mirza and S.A.M. Gilani, "Segmentation of Lungs from CT Scan Images for Eatly Diagnosis of Lung Cancer," Proceedings of World Academy of Science, Engineering and Technology, vol 14, August 2006.

[2] J. S. Silva, "A fast approach to lung segmentation in X-ray CT images”, Proc. Portuguese Conference on Pattern Recognition, 2000, Pp. 415-418.

[3] L. W. Hedlund, R. F. Anderson, P. L. Goulding, J. W. Beck, E. L. Effmann, and C. E. Putman, "Two methods for isolating the lung area of a CT scan for density information," Radiology, vol. 144, pp. 353-357, 1982.

[4] J. M. Keller, F. M. Edwards, and R. Rundle, "Automatic outlining of regions on CT scans," J. Comput. Assist. Tomogr., vol. 5, no. 2, pp. 240-245, 1981.

[5] Y. Y. Boykov and MP. Jolly, "Interactive graph cuts for optimal boundary and region segmentation of objects in N-D images," Int Conf Comput Vision (ICCV'01), 1: 105-112, 2001.

[6] Y. Boykov and G. Funka-Lea, "Graph cuts and efficient N-D image segmentation,” Int J Comput Vision, 70(2): 109-131, 2006.

[7] W. A. Kalender, H. Fichte, W. Bautz, and M. Skalej, "Semiautomatic evaluation procedures for quantitative CT of the lung," J. Comput. Assit. Tomogr., vol. 15, no. 2, pp. 248-255, 1991.

[8] M. S. Brown, M. F. McNitt-Gary, N. J. Mankovich, J. G. Goldin, J. Hiller, L. S. Wilson, and D. R. Aberle, "Method for segmenting chest CT image data using an anatomical model: Preliminary results," IEEE Trans. Med. Imag., vol. 16, pp. 828-839, Dec. 1997. 